\title{
A silkworm hemolymph protein is a prophenoloxidase activation blocker*
}

\author{
Bilang Liu, Xiwu Qi, Qi Han, Ling Jia, Zhonghuai Xiang, Ningjia He \\ State Key Laboratory of Silkworm Genome Biology, Southwest University, Chongqing, China \\ Email: bilangliu@gmail.com, qixiwu@126.com, davy_hq@hotmail.com, jialing132@163.com,xbxzh@swu.edu.cn, \\ "hejia@swu.edu.cn
}

Received 11 August 2012; revised 17 September 2012; accepted 30 September 2012

\begin{abstract}
Melanization in insect hemolymph is triggered by the recognition of pathogen-associated molecular patterns via pattern recognition receptors. The signal transduction leads to the activation of the prophenoloxidase and hence the generation of melanin. The proPO activation process must be tightly controlled to minimize the host damage caused by reactive intermediates during melanin synthesis. The full-length cDNA sequence of a $20 \mathrm{kDa}$ hemolymph protein from Bombyx (Bmhp20) was determined. Bmhp20 gene was expressed in larval fat body, integument, trachea, and ovary and was induced by the challenge of $B$. bombyseptieus. Binding of recombinant Bmhp20 to microbial cell wall components as well as gram-positive bacteria and fungi was confirmed. Phenoloxidase activity assay indicated that recombinant Bmhp20 blocked the proPO activation in hemolymph that was triggered by peptidoglycan or beta-1, 3-glucan. Our data suggest that Bmhp20 plays bifunctional roles in silkworm humoral responses: to participate in pattern recognition and to block the activation of proPO.
\end{abstract}

Keywords: Bombyx mori; Humoral Responses; Pattern Recognition Receptors; Phenoloxidase Activity

\section{INTRODUCTION}

Humoral responses of immunity system in insect such as melanization and induced production of plasma proteins are essential for the host to fight against the pathogens $[1,2]$. For its large body and easily obtained hemolymph, silk- worm is one of the earliest insects used to study humoral responses [3,4].

Pathogen-associated molecular patterns (PAMPs) are conserved structures present on the surfaces of microbes including peptidoglycan (PGN), beta-1, 3-glucan, lipoteichoic acid and lipopolysaccharide [5]. The humoral

\footnotetext{
*The authors declare that they have no competing interests.

${ }^{\#}$ Corresponding author.
}

responses of silkworm are triggered by the recognition of PAMPs via pattern recognition receptors (PRRs) [6]. Yoshida and Ashida (1986) purified a beta-1, 3-glucan recognition protein in silkworm plasma, which can stimulate the activation of the prophenoloxidase (proPO) cascade [3]. Characterization of silkworm peptidoglycan recognition protein (PGRP) indicated that in the absence of PGRP, the proPO cascade could not be triggered by peptidoglycan [7]. Activation of proPO in insects by a cascade of proteolytic cleavages results in the generation of a phenoloxidase (PO). PO oxidizes phenolic compounds to produce quinines that polymerize to form melanin $[8,9]$. Insects used melanin to encapsulate nonself organisms and seal wounds. Since reactive intermediates for melanin synthesis are thought to be harmful to the host insects, the proPO activation process must be tightly controlled to minimize the damage of the host by melanin. Previous studies showed that the activation of proPO system is regulated by serine protease inhibitors and PO inhibitors [10-13].

The availability of silkworm genome makes identification of large number of PRR genes. PGRP genes in silkworm are clustered into two groups located on chromosome 1 (PGRP1-5) and 16 (PGRP 8-11) [14]. Three C-type lectins were identified by searching silkworm genome database and their microorganism recognition specificities were analyzed [15]. The expressions of two PGRP short-type proteins (PGRP-S2 and PGRPS5) and four beta-glucan recognition proteins were induced by the oral infection of Bacillus bombysepticus [16]. In silkworm, mRNAs of most of PRRs usually are identified in fat body and epidermis. They are synthesized and secreted into the hemolymph, where the recognition occurred. Larval hemolymph contains a large number of proteins involved in humoral responses including hemolin, prophenoloxidase, PGRP-SA, serine proteinase-like proteins, and protease inhibitors $[17,18]$. In our previous studies, when we tried to isolate the proteins interacting with a protease inhibitor, we obtained a hemolymph protein (Bmhp20) and its amino terminal sequence was determined [19]. 
The present research is focused on the characterization of this silkworm hemolymph protein. Its full-length cDNA sequence was obtained. We found that the deduced amino acid sequences of Bmhp20 are rich in putative glycosaminoglycan attachment sites. The expression of Bmhp20 gene was enhanced by B. bombyseptieus. In vitro binding assays revealed that recombinant Bmhp20 bound to gram-positive bacteria and fungi. The result of phenoloxidase activity assay indicated that the recombinant Bmhp20 blocked the proPO activation in hemolymph that was activated by PGN or $\beta$-1, 3-glucan.

\section{EXPERIMENTAL PROCEDURES}

\subsection{Silkworm Larvae and Regents}

Silkworm strain p50 is maintained at the State Key Laboratory of Silkworm Genome Biology, Southwest University, China. The larvae are reared on mulberry leaves at $25^{\circ} \mathrm{C}$. The curdlan (Sigma, USA), cellulose (Sigma, USA), and chitin (NEB, USA) were purchased and separately suspended in $100 \mu \mathrm{l}$ PBS (pH 7.4, $200 \mathrm{mM} \mathrm{NaCl}$ ).

\subsection{Database Searching, Gene Cloning, and 5' RACE}

The amino acid sequence data from Edman degradation analysis were blasted against the protein database at SilkDB (http://silkworm.swu.edu.cn/silkdb/). The identified protein sequence and its corresponding nucleic acid sequences (predicted gene BGIBMGA002175) were used as references for primer design.

Total RNA was extracted from the fat body of the fifth instar day 3 larvae using Trizol reagent (Invitrogen, China). RNA samples were treated with DNase I (DNAfree, Ambion) at $37^{\circ} \mathrm{C}$ for $1 \mathrm{~h}$ to remove the DNA contamination. The concentration of RNA was measured in a spectrophotometer (Amersham Biosciences, Sweden). Three micrograms of total RNAs were used to synthesize first-strand cDNA in $25 \mu$ l of reverse transcription PCR (RT-PCR) system with M-MLV Reverse Transcriptase (Promega, USA).

Two primers (forward:

5'-ATGGCACTGAAAATTACTT-3'; reverse: 5'-GGAGCTCTAAGCTGTTAAA-3') were synthesized (Genscript, China) for PCR amplification using the fat body cDNA. PCRs were performed as follows: $94^{\circ} \mathrm{C}$ for $3 \mathrm{~min}, 35$ cycles at $94^{\circ} \mathrm{C}$ for $40 \mathrm{~s}, 55^{\circ} \mathrm{C}$ for $40 \mathrm{~s}, 72^{\circ} \mathrm{C}$ for $40 \mathrm{~s}$, followed by incubation at $72^{\circ} \mathrm{C}$ for $10 \mathrm{~min}$. PCR products were cloned into pMD19-T simple vector (TaKaRa, China). According to the manual of 5' RACE system Version 2.0 (Invitrogen, China), the partial sequenced sequence were used to design the primers GSP1 (5'-GTGTCCAAATCCAGAATCGTG-3'), GSP2 (5'CGAACCCAGAGTCACGACCGAA-3'), and nested primer (5'-AACTGCCGAAGCCGCTATGA-3'). The first-strand gene specific cDNA was reverse-transcribed from fat body RNA by GSP1 using SuperScript ${ }^{\mathrm{TM}}$ II (Invitrogen, China). The specific cDNA was tailed with poly C. Tailed cDNA was used as template to reamplify the target gene using Abridged Anchor Primer 1 (5'-GGCCACGCGTCGACTACGGGGGGGGGG-3') and GSP2. The product was used for the third round PCR amplification using Abridged Universal Amplification Primer 2 (5'-GGCCACGCGTCGACTAGTAC-3') and nested primer. The amplified nucleic acid fragment was cloned into pMD19-T simple vector (TaKaRa, China) and sequenced.

\subsection{Gene Structure and Sequence Analysis}

Gene translation and the prediction of molecular weight and theoretical pI were performed with ExPASy

(http://www.expasy.ch/). Gene structure was drawn by SIM4

(http://pbil.univ-lyon1.fr/members/duret/cours/inserm21

0604/exercise4/sim4.html). Sequence homologous was analyzed on NCBI. Signal peptide prediction was carried out using the program SignalP 3.0

(http://www.cbs.dtu.dk/services/SignalP/). Functional sites in protein were predicted using ELM database (http://elm.eu.org/). TESS

(http://www.cbil.upenn.edu/cgi-bin/tess/tess) was used to predict the Upstream regulatory sequences.

\subsection{RNA Extraction and RT-PCR}

To investigate the expression of Bmhp20 mRNA in tissues, the RNAs from ten different tissues (including fat body, integument, ovary, trachea, head, testis, silk gland, midgut, hemocyte, and malpighian tubule) of the fifth instar day 3 larvae were extracted. For developmental expression profile of Bmhp20, RNAs were prepared using whole larval body from the 1st to the 4th instar larvae and the fat body excised from the fifth instar larva day 1 to adult. RNAs isolation and digestion were carried out as mentioned above. The amplification of the BmActin3 served as an internal control. The Bmhp20 primers (forward: 5'-TTTGGAAATCGCAACCCT-3', reverse: 5'-GATACCGCCCAATGAAGTG-3') and BmActin3 primers (forward:

5'-AACACCCCGTCCTGCTCACTG-3', reverse:

5'-GGGCGAGACGTGTGATTTCCT-3') were used for RT-PCR. PCRs were performed for one cycle at $94^{\circ} \mathrm{C}$ for $3 \mathrm{~min}, 25$ cycles at $94^{\circ} \mathrm{C}$ for $40 \mathrm{~s} ; 55^{\circ} \mathrm{C}$ for $40 \mathrm{~s} ; 72^{\circ} \mathrm{C}$ for $40 \mathrm{~s}$; followed by incubation at $72^{\circ} \mathrm{C}$ for $10 \mathrm{~min}$.

\subsection{Expression, Purification of Recombinant Bmhp20, and Production of Antiserum}

The cDNA fragment encoding the mature Bmhp20 was 
amplified using the fat body cDNA by the following primers: forward:

5'-CTAgctagcTTCCCTCATGGCAGTAGCG-3', reverse: 5'-CCgagctcGTGTTGACATGCCTAGATCAGTGT-3', and then cloned into pMD19-T simple vector (TaKaRa, China) for sequencing. The recombinant plasmid of $\mathrm{pET}$ 28a-Bmhp20 was constructed. One Rosetta colony containing pET28a-Bmhp20 was incubated at $37^{\circ} \mathrm{C}$ in 400 $\mathrm{ml}$ of LB medium supplemented with $25 \mu \mathrm{g} / \mathrm{ml}$ kanamycin until the OD600 reached $0.4-0.8$. A final concentration of $1 \mathrm{mM}$ IPTG was added into the culture and then induced for $3 \mathrm{~h}$ at $37^{\circ} \mathrm{C}$. Cells were harvested by centrifugation. All the following purification procedures were conducted at $4^{\circ} \mathrm{C}$ unless otherwise noted. Cells were washed for 3 times with $20 \mathrm{mM}$ PBS buffer (pH 7.4, $500 \mathrm{mM} \mathrm{NaCl}$ ), and then suspended in $20 \mathrm{ml}$ of same PBS buffer. Cells were disrupted by sonication on ice and separated by centrifugation for $30 \mathrm{~min}$ at $16,000 \times \mathrm{g}$. The supernatant was loaded onto a Ni-NTA affinity agarose column (Invitrogen, China). The fractions eluted with $20 \mathrm{mM}$ PBS (pH 7.4, $500 \mathrm{mM} \mathrm{NaCl}$ ) containing $150 \mathrm{mM}$ imidazole were collected. A gel filtration chromatography was subsequently performed using a Superdex 75 column (AKTA purify). The column was washed with $50 \mathrm{mM}$ PBS (pH 7.4, $200 \mathrm{mM} \mathrm{NaCl}$ ) at a lined linear velocity of $1 \mathrm{ml} / \mathrm{min}$. The fractions containing HisBmhp20 were combined and the proteins were digested by thrombin (Novagen, USA). After the cleavage reaction, biotinylated thrombin was removed with Streptavidin Agarose, and then concentrated by ultrafilter (Amicon Ultra-15 Millipore, USA). The protein concentration was determined by the Bradford protein assay and the bovine serum albumin (BSA) was used as a standard. The cleaved Bmhp20 served as antigen for the production of polyclonal rabbit antiserum. A total of $500 \mu \mathrm{g}$ of cleaved Bmhp20 was equally mixed with complete Freund's adjuvant (Sigma, USA). The resultant emulsified mixture was injected into rabbit. Subsequent booster injections in the rabbit were carried out for three times (250 $\mu$ g per time, once a week). Seven days after the last injection, the rabbit was bled and the blood sample was collected. The antiserum was purified by MAbTrap ${ }^{\mathrm{TM}}$ Kit (GE, USA).

\subsection{Western Blotting Analysis}

The tissues of the fifth instar day 3 larvae were excised and dissolved in 2\% SDS solution. The proteins concentration was determined with BCA kit (Beyotime, China). Two micrograms of each sample was loaded on $15 \%$ SDS-PAGE gel $(2 \mu \mathrm{g} /$ each well). After electrophoresis, proteins were transferred to a PVDF membrane $(0.45 \mu \mathrm{m}$ Roche, Switzerland). Membranes were blocked with 5\% nonfat milk dissolved in Tris-buffered saline Tween-20 (TBST), and then incubated with a 10,000-fold dilution of rabbit-Bmhp20 serum. Immunoreactive bands were detected with anti-rabbit IgG peroxidase (HRP)-conjugated secondary antibodies (1:10,000, Sigma, USA). The signals were visualized using the ECL Plus Western blotting detection reagent (GE, USA).

\subsection{Microbial Challenge and Real Time PCR}

The fifth instar day 3 larvae were injected with the formaldehyde-fixed E. coli $\left(10^{8}\right.$ cells per larvae), B. bombyseptieu $\left(10^{7}\right.$ cells per larvae), B. bassiana $\left(10^{7}\right.$ cells per larvae), respectively. Injection of $10 \mu \mathrm{l}$ of $0.85 \% \mathrm{NaCl}$ was used as a negative control. Fat body from challenged larvae was obtained 1, 3, 6, 12, $24 \mathrm{~h}$ after injection. The RNAs were isolated and first-strand cDNAs were synthesized as mentioned above. For real-time PCR, primers of Bmhp20 (forward:

5'-AGTAGCACAAGCCTTCCCTCATG-3'; reverse: 5'-ACTGCCGAAGCCGCTATGAC-3') and primers of sw22934 (forward:

5'-TTCGTACTGGCTCTTCTCGT-3'; reverse:

5'-CAAAGTTGATAGCAATTCCCT - 3') were designed. The primer annealing temperatures was $60^{\circ} \mathrm{C}$ and the size of product was $139 \mathrm{bp}$. Primer specificity was visualized by $2 \%$ agarose gel. The PCR efficiency was within 95\% - 110\%. The real-time PCRs were performed using the SYBR Premix Ex Taq kit (TaKaRa, China). Each reaction in $25 \mu \mathrm{l}$ reaction cocktail including $12.5 \mu \mathrm{l}$ of Ex Taq, $70 \mathrm{ng}$ cDNA, $10 \mathrm{mM}$ Bmhp20 forward and reverse primers. The PCR reaction was performed on an ABI Prism 7000 Sequence Detection System (Applied Biosystems) using the following program: initial denaturation at $95^{\circ} \mathrm{C}$ for $10 \mathrm{~s}$, and 40 cycles of $95^{\circ} \mathrm{C}$ for $5 \mathrm{~s}$, $60^{\circ} \mathrm{C}$ for $31 \mathrm{~s}$. The primers of sw22934 (transcription initiation factor 2 gene) was used as internal standardizetion.

\subsection{In Vitro Binding Assay of Bmhp20 with Microbial Cell Wall Components and Microbes}

In vitro binding assays were executed according to published protocol [20]. Six kinds of log phase microbes (3 $\mathrm{ml}), S$. aureu, B. bombyseptieus, $S$. marcescens, $P$. aerugi$n o s a, B$. bassiana, and $P$. pastoris were collected and fixed with $2 \%$ formaldehyde for $2 \mathrm{~h}$. The cells were washed by PBS buffer (pH 7.4, $200 \mathrm{mM} \mathrm{NaCl}$ ) and suspended in $100 \mu \mathrm{l}$ of the same PBS. In addition, $2 \mathrm{mg}$ of curdlan, cellulose, and were suspended in $100 \mu \mathrm{l}$ PBS (pH 7.4, $200 \mathrm{mM} \mathrm{NaCl).} \mathrm{Two} \mathrm{micrograms} \mathrm{of} \mathrm{Bmhp20}$ was then added to the above cell/polysaccharide solution and incubated for $2 \mathrm{~h}$ at room temperature. After centrifugation, the pellets were washed for five times with PBS buffer. The remaining pellets were resuspended in $100 \mu \mathrm{l}$ PBS buffer and boiled in SDS sample buffer for 5 
min. The proteins detached from the pellets were analyzed by Western blotting.

\subsection{In Vitro Phenoloxidase Activity Assay}

Silkworm larva plasma (SLP) reagent (Wako, Japan) was used to measure the phenoloxidase (PO) activity. Two types of polysaccharide, PGN (53243 Sigma, USA) extracted from M. luteus and laminarin (L9634 Sigma, USA), a kind of $\beta$-1,3-glucan extracted from $L$. digitata, were used as inducers. The assays were performed as described in manufacturer's instructions. In brief, $20 \mu \mathrm{l}$ recombinant Bmhp20 (1.5 $\mu \mathrm{g} / \mu \mathrm{l}), 20 \mu \mathrm{l}$ PGN $(0.2 \mathrm{ng} / \mu \mathrm{l})$ or $20 \mu \mathrm{l}$ laminarin $(0.2 \mathrm{ng} / \mu \mathrm{l})$, and $10 \mu \mathrm{l}$ double distilled water $\left(\mathrm{ddH}_{2} \mathrm{O}\right)$ were mixed together. After incubated at $27^{\circ} \mathrm{C}$ for $5 \mathrm{~min}, 50 \mu \mathrm{l}$ of SLP reagent was added. Then the mixture was incubated for another $30 \mathrm{~min}$ at $27^{\circ} \mathrm{C}$. PO activity was monitored at absorbance of $450 \mathrm{~nm}$. The assay was repeated three times.

\section{RESULTS AND DISCUSSION}

\subsection{Full-Length cDNA and Sequence Analysis of Bmhp20}

The sequence of 20 residues

"HGSSDVDGSGEVEAVAGTLK" was used to search the silkDB (http://silkworm.swu.edu.cn/silkdb/) with TBLASTN program. A protein (BGIBMGA002175) with the same deduced amino acid sequence was found. However, the 5'cDNA sequence of this gene is absent. We then performed 5'RACE to clone its full length cDNA. As a result, a 740-bp cDNA sequence was obtained, as shown in Figure 1(a). This gene has an open reading frame of $648 \mathrm{bp}$, encoding a 215-residue polypeptide with an 18-residue signal peptide predicted by SignalP 3.0. The mature protein has a calculated molecular weight of $21.6 \mathrm{kDa}$ and a $\mathrm{pI}$ of 5.5. This protein was so named as Bombyx mori hemolymph protein 20 (Bmhp20, access number: GU015849) based on its size and presence in hemolymph. Also shown in Figure 1(a), a predicted transcription start site A was found 13 nt upstream of the ATG codon. A polyadenylation sequence AATAAA was observed at the end of Bmhp20 mRNA.

Comparison of the cDNA and its genome sequences revealed that Bmhp20 had three exons separated by two introns (Figure 1(b)). The first exon consists of 13 bp 5' untranslated region and partial coding sequence of the signal peptide. The second exon encodes residues 5 - 17 of the signal peptide. The last exon codes for the remaining residue of signal peptide and the mature protein of Bmhp20. It was found that Bmhp20 had only one copy in silkworm genome which was located on chromosome 15.

The deduced amino acid sequences of Bmhp20 were shown in Figure 1(c). The amino acid composition of
Bmhp20 has a low complexity, which is rich in glycine (26.9\%), serine (13.2\%), phenylalanine (12.2\%), aspartate acid $(10.7 \%)$, and histidine $(9.1 \%)$. It is worth to mention that Bmhp20 is rich of glycosaminoglycan attachment sites (DGSGE, DNSGF, NSGF, HSGF, DSGF, and DSAL) predicted by ELM [21], as shown in Figure 1(c).

\subsection{The Temporal and Spatial Expression Profiles of Bmhp20 Gene}

RT-PCR was carried out using RNAs extracted from fat body, integument, ovary, trachea, head, testis, silk gland, midgut, hemocyte, and malpighian tubule of the fifth instar day 3 larvae. As shown in Figure 2(a), the Bmhp20 transcripts were found to be expressed in larval fat body, integumen, trachea, and ovary. Furthermore, the temporal expression profile of Bmhp20 was investigated by RT-PCR. The results in Figure 2(b) showed that Bmhp20 gene was expressed in all of the larval stages. When the larva entered the wandering stage, Bmhp20 gene was highly expressed at the first day and its expression was decreased at W2 and W3 (the second and third day of the wandering stage, respectively). At the pupal stages, the transcripts of Bmhp20 were undetectable from P1 to P3, up-regulated at $\mathrm{P} 4$, and then dramatically decreased from P5 to P7 (P1 - P7, day 1 - 7 of pupa).

\subsection{The Transcriptional Regulation of Bmhp20 against Microbial Challenge}

Real-time RT-PCR was carried out to measure the transcriptional regulation of Bmhp20 in the fat body challenged by B. bombyseptieus, Beauveria bassiana, and Escherichia coli. As illustrated in Figure 2(c), Bmhp20 was rapidly induced by all challenges after injections for $1 \mathrm{~h}$. However, after injection for 3 to $6 \mathrm{~h}$, the expressions of Bmhp20 were leveled off. Interestingly, in contrast to B. Bassiana and E. coli, B. bombyseptieus strongly induced the expression of Bmhp20 (up to 6-fold) after injection for 12 to $24 \mathrm{~h}$. Our data indicated that Bmhp20 might involve in the immune responses of silkworm.

\subsection{Production and Purification of Recombinant Bmhp20}

His-Bmhp20 was expressed in prokaryotic expression system and purified by a two-step chromatography. Histag at the $\mathrm{N}$ terminal of his-Bmhp20 was removed with biotinylated thrombin. As shown in Figure 3, on a SDSPAGE, the band of purified recombinant Bmhp20 without his-tag migrated with a molecular size around $26 \mathrm{kDa}$ which is different from the putative molecular weight of Bmhp20. We assumed that the delayed migration of Bmhp20 on SDS-PAGE may result from that the SDSstable structures of Bmhp20. 


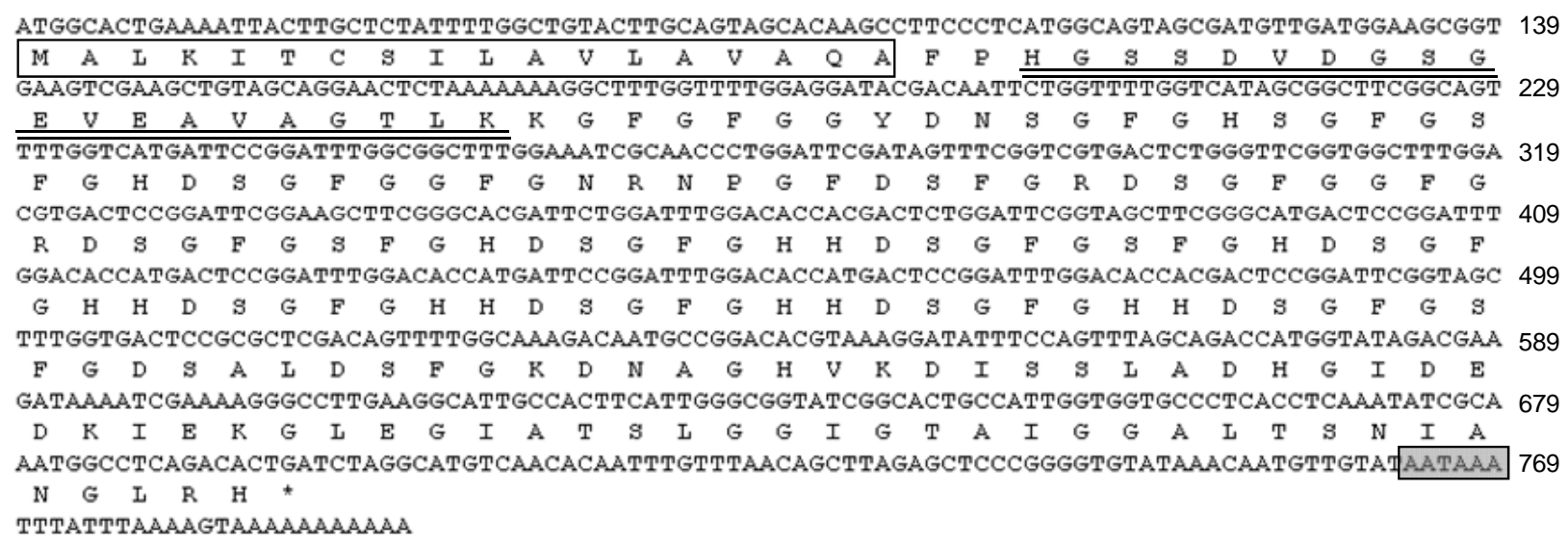

(a)

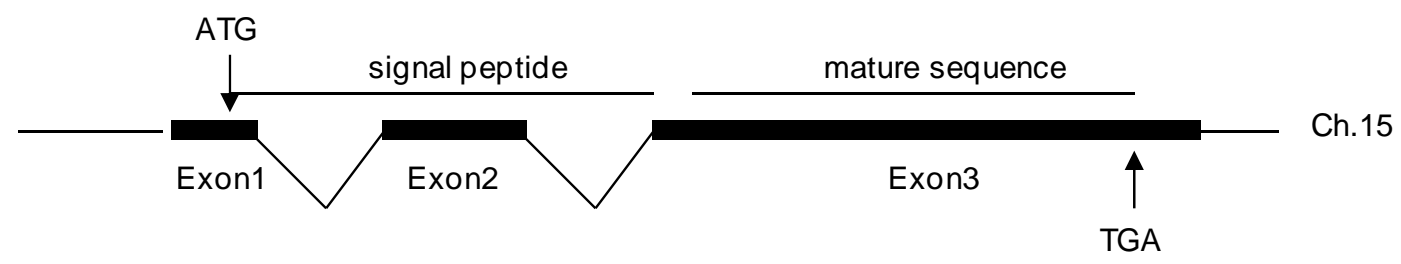

(b)

\begin{abstract}
HGSSDVDGSGEVEAVAGTLKKGFGFGGYDNSGFGHSGFGSFGHDSGFGGFGNRNPGFDSFGRDSGFGGFGRD SGFGSFGHDSGFGHHDSGFGSFGHDSGFGHHDSGFGHHDSGFGHHDSGFGHHDSGFGSFGDSALDSFGKDNA GHVKDISSLADHGIDEDKIEKGLEGIATSLGGIGTAIGGALTSNIANGLRH
\end{abstract}

(c)

Figure 1. Gene sequence of Bmhp20 and its deduced amino acid sequence. (a) The cDNA sequence of Bmhp20 protein. The deduced amino acid sequence is shown below the cDNA sequence. The transcriptional start site of A is indicated by an arrow and is designated +1 . The polyadenylation sequence AATAAA is in grey frame. Predicted signal peptide is boxed and the $\mathrm{N}$-terminal sequence determined by Edman degradation is double-underlined; (b) Genomic structure of Bmhp20 gene. Black boxes represent exons; (c) The deduced amino acid sequence of Bmhp20 mature protein. The predicted glycosaminoglycan attachment sites are shown in boldface italic.

\subsection{Bmhp20 Is Secreted into Hemolymph}

The distribution of Bmhp20 in different tissues was examined by Western blotting. Same amount of proteins were separated by SDS-PAGE and stained by silver as a loading control (Figure 4(b)). On the membrane, as shown in Figure 4(a), only one band in hemolymph was detected, indicating that the mature Bmhp20 was secreted into hemolymph. The Bmhp20 was previously annotated as a cuticular protein (CPRG16, accession number: NM_001167717) for its rich of glycine and owning a GGX/AAP repeat sequence [22]. The result of Western blotting assay obviously showed that Bmhp20 is a hemolymph protein, rather than a structural protein present in the silkworm cuticles.

\subsection{Recombinant Bmhp20 Binds to Various Microorganisms in Vitro}

As mentioned above, the analysis of the amino acid se- quence of Bmhp20 revealed that it has abundant putative glycosaminoglycan attachment sites (Figure 1(c)). We assumed that Bmhp20 might be involved in the pattern recognition. Therefore we investigated the binding activity of Bmhp20 by three kinds of microbial cell wall components (Figure 5(a)). Bmhp20 was incubated with these polysaccharides separately. After washing for five times with PBS, the proteins eluted from polysaccharides were detected by Western blotting. The results showed that Bmhp20 was bound to chitin, cellulose, and curdlan. In vitro binding assays of recombinant Bmhp20 were thereafter performed with gram-positive bacteria (Staphylococcus aureus and $B$. bombyseptieus), gram-negative bacteria (Serratia marcescens, and Pseudomonas aerugi$n o s a$ ), and fungi (B. bassiana and Pichia pastoris). As shown in Figure 5(b), Bmhp20 bound to $S$. aureus, $B$. bombyseptieus, B. bassiana, and P. pastoris. Recombinant Bmhp20 also had weak binding activity to $S$. marcescens and there was no protein eluted from P. aeruginosa, 


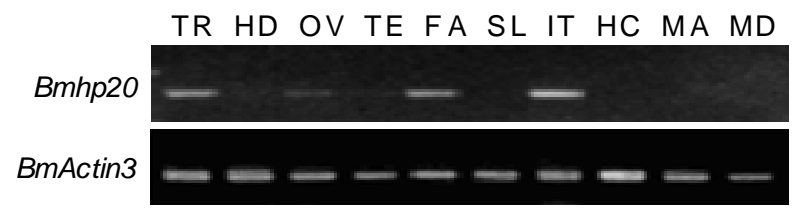

(a)

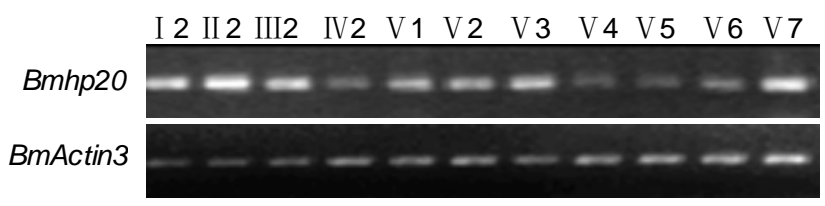

W1 W2 W3 P1 P2 P3 P4 P5 P6 P7 A1

(b)

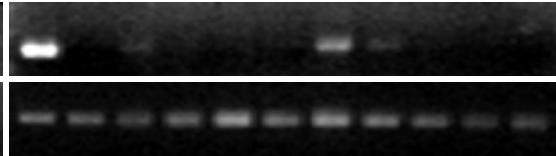

Bmp20

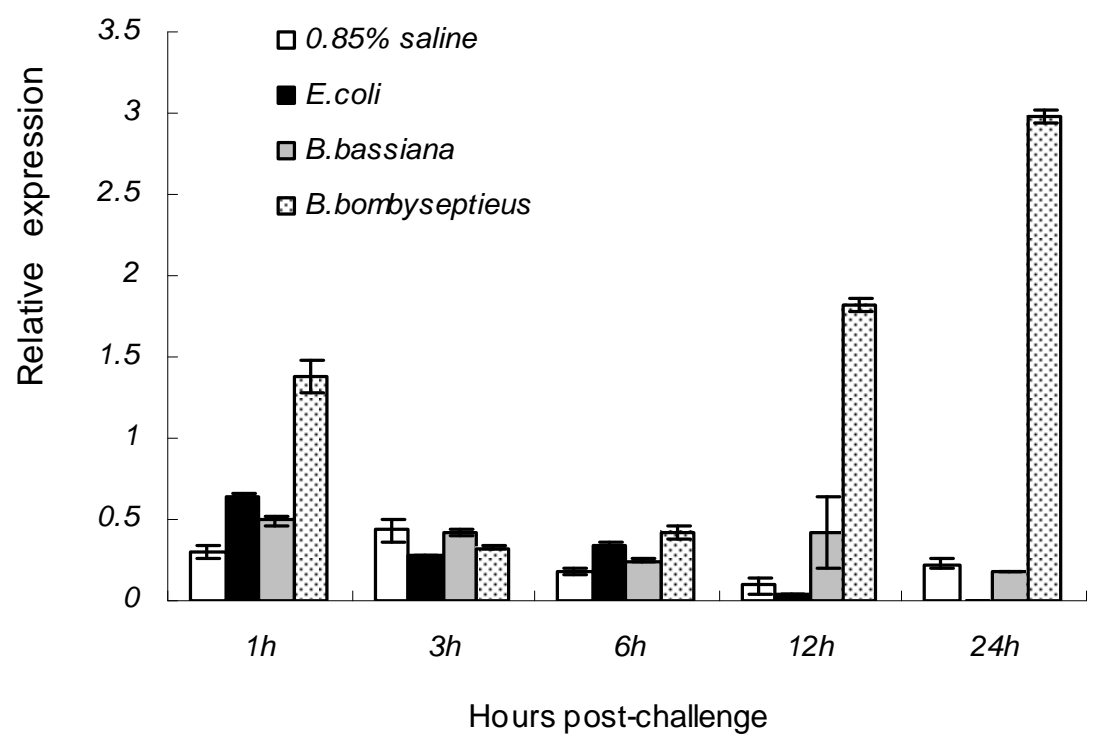

(c)

Figure 2. The expression of Bmhp20 gene. The spatial (a) and temporal (b) expression profiles of Bmhp20 were performed by RT-PCR as described in Material and Methods. (a) The tissue distribution of Bmhp20 in silkworm larvae at fifth instar day 3 including trachea (TR), head (HD), ovary (OV), testis (TE), fat body (FA), silk gland (SL), integument (IT), hemocyte (HC), malpighian tubule (MA), and midgut (MD); (b) The developmental expression profile of Bmhp20 gene from the first instar larvae, day 2 (I2) to adult day 1 (A1). I2 stands for day 2 of the 1st instar larvae, II2 day 2 of the 2nd, III2 and IV2 day 2 of the 3rd and 4th, respectively. V1 to V7, day 1 - 7 of the 5 th instar larvae. W1-W3, day 1-3 of the wandering phase. P1 to P7, day 1 7 of the pupa. A1, day 1 of the adult; (c) Quantitative real-time PCR analysis of Bmhp20 mRNA in fat body after injection of E. coli, B. bassiana and B. bombyseptieus at the indicated time points. The error bars represent the standard error of the mean $(n=3)$.

suggesting little binding of Bmhp20 to this gram-negative bacterium.

\subsection{Recombinant Bmhp20 Inhibits the PO Activity That Is Activated by Microbial Cell Wall in Vitro}

PGN or $\beta$-glucan activates the PO cascade of silkworm larval plasma. The produced melanin can be easily detected with a substrate 3, 4-dihydroxyphenylalanine [23]. Silkworm larva plasma (SLP) reagent has been used for the detection of PGN in human plasma (Kobayashi et al.,
2000). Since Bmhp20 bound to Gram-positive bacteria and fungi cell walls, we then used the SLP reagent to examine whether Bmhp20 was involved in the activation of PO cascade. The activated PO activeity was monitored at absorbance of $450 \mathrm{~nm}$ after the SLP reagents incubated with the test samples at $27^{\circ} \mathrm{C}$ for $30 \mathrm{~min}$. As shown in Figure 6, compared to SLP alone, when PGN or laminarin was added into the SLP, the PO activities dramatically increased. In the case of his-Bmhp20, the PO activity was not changed by recombinant protein itself. In this context, when PGN or laminarin was added into 


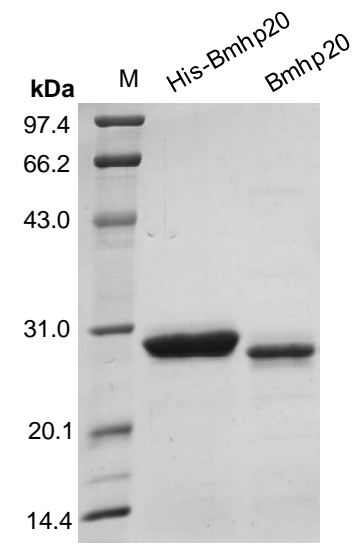

Figure 3. The production of the recombinant Bmhp20 protein. Purified recombinant his-Bmhp20 and Bmhp20 without his tag were analyzed by $15 \%$ SDS-PAGE and the gel was stained with Coomassie Brilliant Blue. the SLP reagent pre-incubated with his-Bmhp20, the PO activities were no longer increased, indicating that Bmhp20 acts as a proPO activation blocker. Previous studies of proPO-activating proteinases in silkworm revealed at least two enzymes, i.e. an esterase hydrolyzing $\mathrm{N}$ - $\alpha$ benzoyl-L-arginine ethyl ester (BAEEase), a prophenoloxidase activating enzyme (PPAE) were involved in the activation pathway $[25,26]$. BAEEase activity was shown to be inactivated by silkworm antitrypsin, whereas the activation of PPAE was unaffected by protease inhibitors [27]. Phenoloxidase inhibitor (POI) was isolated from the housefly Musca domesticca and Manduca sexta [12,13]. Bmhp20 has little sequence similarity with above protease inhibitors and low molecular weight peptides. Binding to microbial surfaces and blocking the proPO activation make Bmhp20 a distinct hemolymph protein involved in the silkworm humoral responses.
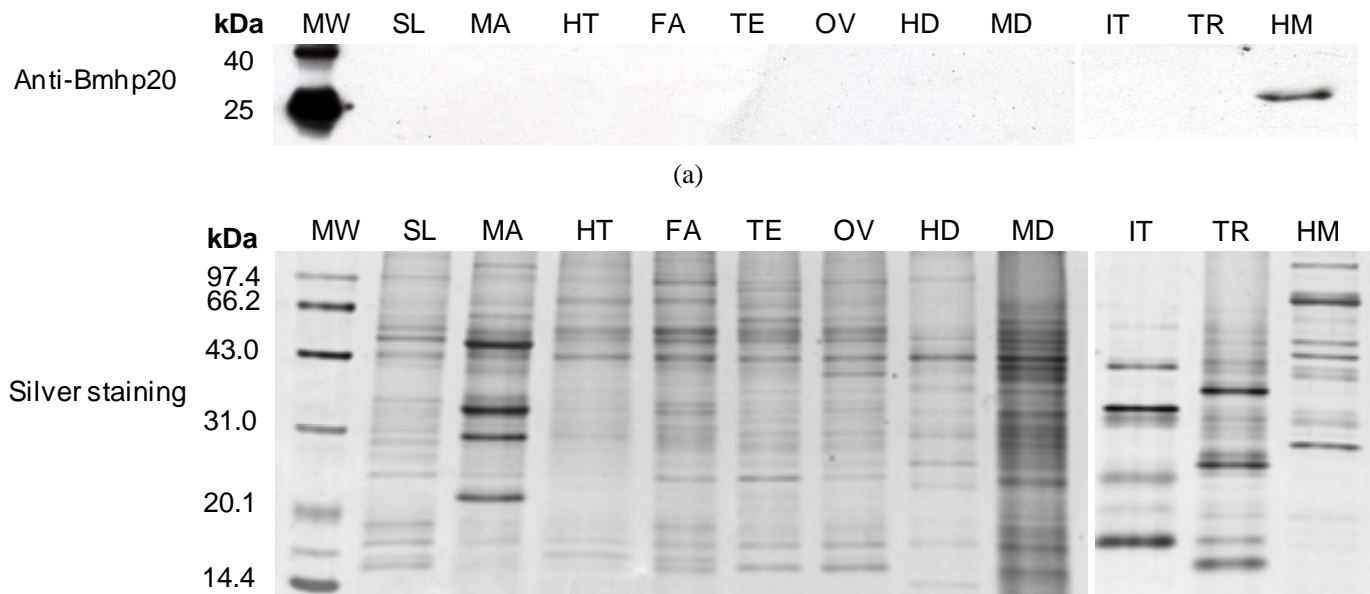

(b)

Figure 4. The expression of Bmhp20 protein. Western blotting analysis of the tissue distribution of Bmhp20 in the fifth instar day 3 silkworm larvae. The proteins were isolated from the trachea (TR), head (HD), ovary (OV), testis (TE), fat body (FA), silk gland (SL), integument (IT), heymocyte (HC), malpighian tubule (MA), hemolymph (HM), and midgut (MD).

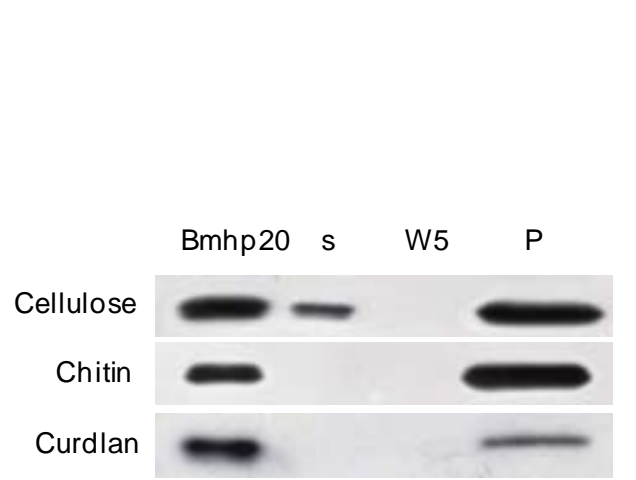

(a)

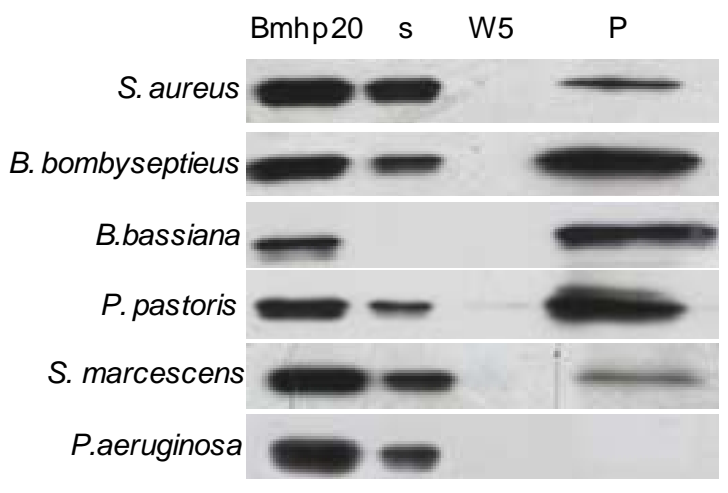

(b)

Figure 5. In vitro binding assay of Bmhp20 protein with various polysaccharides and microbes. (a) Binding assay of Bmhp20 with cellulose, chitin, and curdlan; (b) Binding assay of Bmhp20 to gram-positive bacteria ( $S$. aureus, B. bombyseptieus), gram-negative bacteria (S. marcescens, P. aeruginos), and fungi (B. bassiana, P. pastoris). The bands were detected by Western blotting. S: supernatant; W5: fraction from the fifth wash; P: pellet. 


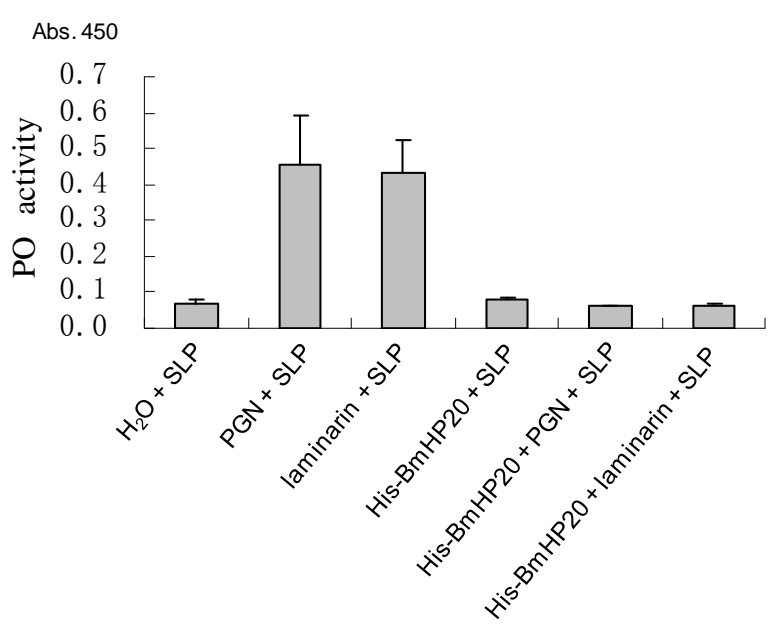

Figure 6. Recombinant Bmhp20 inhibits the PO activity that is activated by PGN or laminarin. $\mathrm{H}_{2} \mathrm{O}$, PGN (4 ng), laminarin (4 ng), His-Bmhp20 (30 $\mu$ g), PGN (pre-incubated with $30 \mu \mathrm{g}$ His-Bmhp20 for 5 min at $27^{\circ} \mathrm{C}$ ) and laminarin (4 ng) (pre-incubated with $30 \mu \mathrm{g}$ His-Bmhp20 for $5 \mathrm{~min}$ at $27^{\circ} \mathrm{C}$ ) were incubated with $50 \mu \mathrm{SLP}$ reagent for $30 \mathrm{~min}$ at $27^{\circ} \mathrm{C}$. The absorption of each sample was monitored at 450 $\mathrm{nm}$. Each assay was replicated three times. The bars represent the mean of three individual measurements.

To our knowledge, proteins with recognition activities have not yet been reported to inhibit the proPO-activating system of insect. It is concluded that for the first time, we identified a silkworm hemolymph protein participating in pattern recognition as well as blocking pro$\mathrm{PO}$ activation.

\section{AUTHORS' CONTRIBUTIONS}

$\mathrm{NH}$ conceived of the study and developed the study design. BL and XQ carried out the analysis and BL drafted the manuscript. QH did the PO activity assay. LJ performed the binding assay. ZX participated in the study design. NH contributed to the critical revision of the manuscript. All authors read and approved the final manuscript.

\section{ACKNOWLEDGEMENTS}

This work was supported by research grants from the National Basic Research Program of China (Grant No. 2012CB114600), Natural Science Foundation Project of CQ CSTC (Grant No. cstc2011jjjq0010), and 111 project (B07045). The nucleotide sequence reported in this paper has been submitted to Genebank ${ }^{\mathrm{TM}}$ with an accession number GU015849.

\section{REFERENCES}

[1] Hoffmann, J.A. and Reichhart, J.M. (2002) Drosophila innate immunity: An evolutionary perspective. Nature Immunology, 3, 121-126. doi:10.1038/ni0202-121
[2] Gillespie, J.P., Kanost, M.R. and Trenczek, T. (1997) Biological mediators of insect immunity. Annual Review of Entomology, 42, 611-643. doi:10.1146/annurev.ento.42.1.611

[3] Yoshida, H., Ochiai, M. and Ashida, M. (1986) Beta-1, 3-glucan receptor and peptidoglycan receptor are present as separate entities within insect prophenoloxidase activating system. Biochemical and Biophysical Research Communications, 141, 1177-1184. doi:10.1016/S0006-291X(86)80168-1

[4] Ochiai, M. and Ashida, M. (1988) Purification of a beta-1, 3-glucan recognition protein in the prophenoloxidase activating system from hemolymph of the silkworm, Bombyx mori. The Journal of Biological Chemistry, 263, 12056-12062.

[5] Janeway, C.A. and Medzhitov, R. (2002) Innate immune recognition. Annual Review of Immunology, 20, 197-216. doi:10.1146/annurev.immunol.20.083001.084359

[6] Mogensen, T.H. (2009) Signaling in innate immune defenses pathogen recognition and inflammatory. Clinical Microbiology and Molecular Biology Reviews, 22, 240. doi:10.1128/CMR.00046-08

[7] Yoshida, H., Kinoshita, K. and Ashida, M. (1996) Purification of a peptidoglycan recognition protein from hemolymph of the silkworm, Bombyx mori. The Journal of Biological Chemistry, 271, 13854-13860. doi:10.1074/jbc.271.23.13854

[8] Söderhäll, K. and Cerenius, L. (1998) Role of the prophenoloxidase activating system in invertebrate immunity. Current Opinion in Immunology, 10, 23-28. doi:10.1016/S0952-7915(98)80026-5

[9] González-Santoyo, I. and Córdoba-Aguilar, A. (2011) Phenoloxidase: A key component of the insect immune system. Entomologia Experimentalis et Applicata, 142, 1116. doi:10.1111/j.1570-7458.2011.01187.x

[10] Jiang, R., Kim, E.H, Gong, J.H., et al. (2009) Three Pairs of Protease-Serpin Complexes Cooperatively Regulate the Insect Innate Immune Responses. The Journal of Biological Chemistry, 284, 35652-35658. doi:10.1074/jbc.M109.071001

[11] Ligoxygakis, P., Pelte, N., Leclerc, V., et al. (2002) A serpin mutant links Toll activation to melanization in the host defence of Drosophila. The EMBO Journal, 1, 63306337. doi:10.1093/emboj/cdf661

[12] Lu, Z. and Jiang, H. (2007) Regulation of phenoloxidase activity by high- and low-molecular-weight inhibitors from the larval hemolymph of Manduca sexta. Insect Biochemistry and Molecular Biology, 37, 478-485. doi:10.1016/j.ibmb.2007.02.004

[13] Tsukamoto, T., Ichimaru, Y., Kanegae, N., et al. (1992) Identification and isolation of endogenous insect phenoloxidase inhibitors. Biochemical and Biophysical Research Communications, 184, 86-92. doi:10.1016/0006-291X(92)91161-I

[14] Cheng, T.C., Xia, Q.Y., Xu, P.Z., et al. (2009) Identification and comparative analysis of immune-related genes and signaling pathways in the silkworm, Bombyx mori. Acta Entomologica Sinica, 52, 235-245. 
[15] Takase, H., Watanabe, A., Yoshizawa, Y., et al. (2009) Identification and comparative analysis of three novel C-type lectins from the silkworm with functional implications in pathogen recognition. Developmental \& Comparative Immunology, 33, 789-800. doi:10.1016/j.dci.2009.01.005

[16] Huang, L., Cheng, T., Xu, P., et al. (2009) A genomewide survey for host response of silkworm, Bombyx mori during pathogen Bacillus bombyseptieus infection. PLoS One, 4, e8098. doi:10.1371/journal.pone.0008098

[17] Li, J.Y., Li, J.S. and Zhong B.X. (2012) Proteomic profiling of the hemolymph at the fifth instar of the silkworm Bombyx mori. Insect Science, 19, 441-454.

[18] Hou, Y., Zou, Y, Wang, F., et al. (2010) Comparative analysis of proteome maps of silkworm hemolymph during different developmental stages. Proteome Science, 8, 4554. doi:10.1186/1477-5956-8-45

[19] He, N., Fujii, H., Kusakabe, T., et al. (2004) Over-expression in Escherichia coli and purification of recombinant CI-b1, a Kunitz-type chymotrypsin inhibitor of silkworm. Protein Expression and Purification, 38, 9-16. doi:10.1016/j.pep.2004.07.010

[20] Gandhe, A.S., John, S.H. and Nagaraju, J. (2007) Noduler, a novel immune up-regulated protein mediates nodulation response in insects. The Journal of Immuno$\operatorname{logy}$, 179, 6943-6951.

[21] Gould, C.M., Diella, F., Via, A., et al. (2012) ELM: The status of the 2010 eukaryotic linear motif resource. $\mathrm{Nu}$ cleic Acids Research, 38, 167-180.

\section{LIST OF ABBREVIATIONS}

PAMPs, Pathogen-associated molecular patterns; PRRs, pattern recognition receptors; proPO, prophenoloxidase; PGRP, peptidoglycan recognition protein; PO, phenoloxi-

\section{doi:10.1093/nar/gkp1016}

[22] Futahashi, R., Okamoto, S., Kawasaki, H., et al. (2008) Genome-wide identification of cuticular protein genes in the silkworm, Bombyx mori. Insect Biochemistry and Molecular Biology, 38, 1138-1146. doi:10.1016/j.ibmb.2008.05.007

[23] Tsuchiya, M., Asahi, N., Suzuoki, F., et al. (1996) Detection of peptidoglycan and $\beta$-glucan with silkworm larvae plasma test. FEMS Immunology and Medical Microbiology, 15, 129-134.

[24] Kobayashi, T., Tani, T., Yokota, T., et al. (2000) Detection of peptidoglycan in human plasma using the silkworm larvae plasma test. FEMS Immunology and Medical Microbiology, 28, 49-53. doi:10.1111/j.1574-695X.2000.tb01456.x

[25] Aigaki, T., Kasuga, H. and Osanai, M. (1987) A specific endopeptidase, BAEE esterase, in the glandula prostatica of the male reproductive system of the silkworm, Bombyx mori. Insect Biochemistry and Molecular Biology, 17, 323-328. doi:10.1016/0020-1790(87)90075-8

[26] Satoh, D., Horii, A., Ochiai, M., et al. (1999) Prophenoloxidase-activating enzyme of the silkworm, Bombyx mori. Purification, characterization, and cDNA cloning. The Journal of Biological Chemistry, 274, 7441-7453. doi:10.1074/jbc.274.11.7441

[27] Ashida, M. and Sasaki, T. (1994) A target protease activity of serpins in insect hemolymph. Insect Biochemistry and Molecular Biology, 24, 1037-1041. doi:10.1016/0965-1748(94)90141-4

dase; PGN, peptidoglycan; IPTG, isopropyl $\beta$-D-thiogalactoside; SLP, silkworm larva plasma; BAEEase, N$\alpha$-benzoyl-L-arginine ethyl ester; PPAE, prophenoloxidase activating enzyme. 